\title{
Measurement of the Velocity Profile in the Spirally Reinforced Pipe
}

\author{
Xingfu Zhong ${ }^{1}$, Liming Lin ${ }^{1}$, Zhangjun ${ }^{1}$, Yingxiang $\mathrm{Wu}^{1}$ \\ ${ }^{1}$ Institute of Mechanics, CAS, Beijing,100190, China \\ E-mail: xfzhong@imech.ac.cn
}

Keywords: velocity profile; spirally reinforced grooves; a large-diameter pipe

Abstract. In present paper, by installing the wind tunnel consisted of the spirally-reinforced-groove pipe with the diameter of $325 \mathrm{~mm}$, the velocity at different radial positions is measured in the text section where the flow is fully developed into the turbulent. Correspondingly, the velocity profile is obtained. By comparison with the theoretical velocity profile in the smooth pipe, the measured results have shown that the velocity profile in such special pipe is obviously different; especially at the non-dimensional radial position of 0.45 by the radius of pipe, the local minimum of velocity is appeared. These phenomena indicate that the spirally-reinforced grooves not only affect the variation of velocity profile, but also disturb the instability and accuracy of measurements in a certain extent.

\section{Introduction}

The velocity distribution in large diameter pipe is very important for a measurement of gas flow rate. The spirally reinforced pipe (SRP) is this kind of pipe. It has a large diameter (from $200 \mathrm{~mm}$ to $800 \mathrm{~mm}$ ), can bear high pressure, and is used in the pipeline of chemical, coal and metallurgy industry. In order to get gas flow rate, many kinds of flow meter are used. The conventional approach is measuring the axial velocity in the pipe, and accordingly calculating the flow rate indirectly. It may induce some errors at different Reynolds numbers, because the calculated results are affected by many factors. Up to now these factors taken into account are turbulent flow, Reynolds number, gas density, the large diameter, and wide range of flow rate. Few researchers considered other factors, such as the structure of the pipe itself. It affects the velocity distribution at different positions of the SRP. The velocity distribution is one of the most important factors. This paper discusses the velocity distribution in turbulent flow based on the theoretical analysis and calculation of a lot of data groups measured in the case of different Reynolds numbers.

\section{Experiment setup}

The experimental apparatus is sketched in Figure 1. It consists of a horn mouth, D325 SRP, plexiglass pipe, draught fan and frequency transformer. When the draught fan works on, the air was sucked in the pipeline. The air flow rate was controlled by frequency transformer. Its

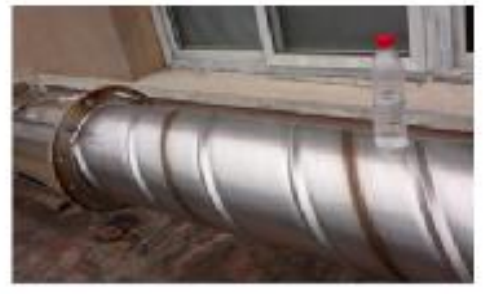

a. The photo of D325 SRP

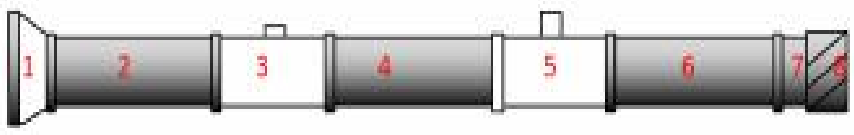

b. The experimental setup.

Fig. 1 Schematic of the experimental setup. 1,7-horn mouth, 2,4,6-D325 SRP, 3,5-plexiglass pipe, 8-draught fan mean flow velocity is from 1 to $12 \mathrm{~m} / \mathrm{s}$. In the experiment, the averaged Reynolds numbers are $2.3 \times 10^{3}$ to $1.9 \times 10^{4}$, according to the definition of Reynolds number. The flow is in a turbulent state when $\operatorname{Re} \geq 2 \times 10^{3}$, and all kinds of experimental conditions are also in a turbulent state. 
The experiment used the pipeline with inner diameter of $325 \mathrm{~mm}$ and 50D long. The test section is the straight part in the middle of pipeline. The local velocities of different position along with diameter were measured by Hot Wire Anemometer (HWA). Its accuracy is $1 \%$ from 0 to $30 \mathrm{~m} / \mathrm{s}$, and is used in constant-temperature mode of operation. The hot-wire probe installation at pipeline of section 5 shown in Figure 1b. The hot-wire probe can be moved along with diameter between the pipe wall and the center. The measured velocity in the cross section was output with voltage signal.

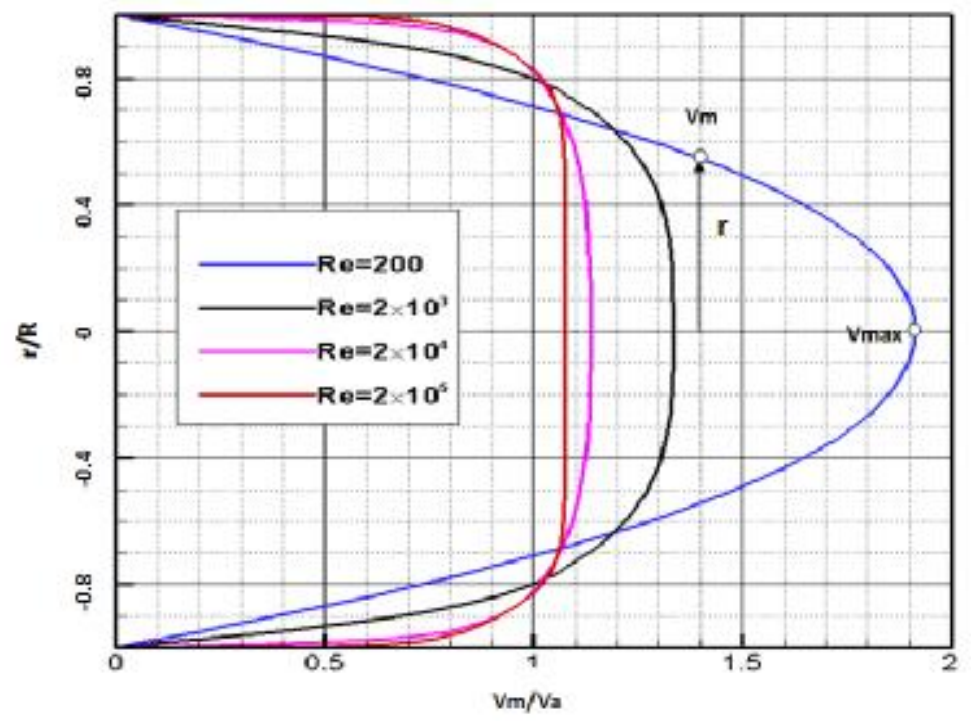

Fig.2 The theoretical flow velocity profile

\section{Theoretical velocity flow profile}

The most commonly used description of flow conditions within the pipe is the flow velocity profile. If gas flows in a circle smooth pipe, pipe radius is $\mathrm{R}$. Va is mean flow velocity in the inlet section, and $\mathrm{Vm}$ is the local velocity at $\mathrm{r}$. The shape of the flow velocity profile is given by the following equation:

$$
\frac{V_{m}}{V_{a}}=\left[1-\frac{r}{R}\right]^{1 / n}
$$

The value of $\mathrm{n}$ determines the shape of the flow velocity profile. It is a function of Reynolds number and friction factor. Figure 2 shows the theoretical flow velocity profile for gas measurement. When $\operatorname{Re} \geq 2 \times 10^{4}$, the flow velocity profile near the center tends to flat.

\section{Measurement results}

Figure 3(a) and Figure 3(b) present the time series of measured velocity acquired by HWA for different gas flow velocity. The time series show that the measured velocities have a violent fluctuation when the gas flow velocity greater. When the gas flow velocity is same, the measured velocity at $r / R=0.138$ is greater than $r / R=0.631$. 


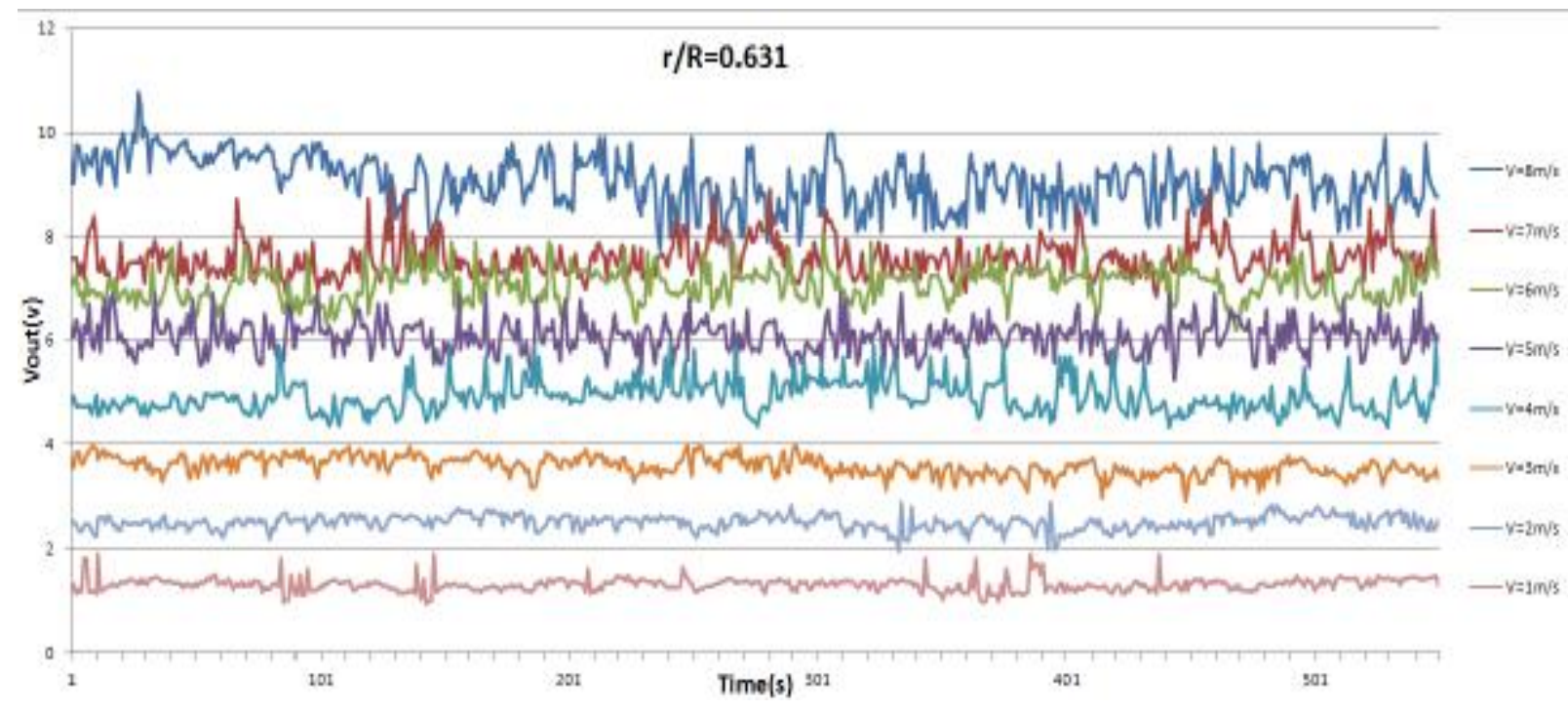

a. $\mathrm{r} / \mathrm{R}=0.631$

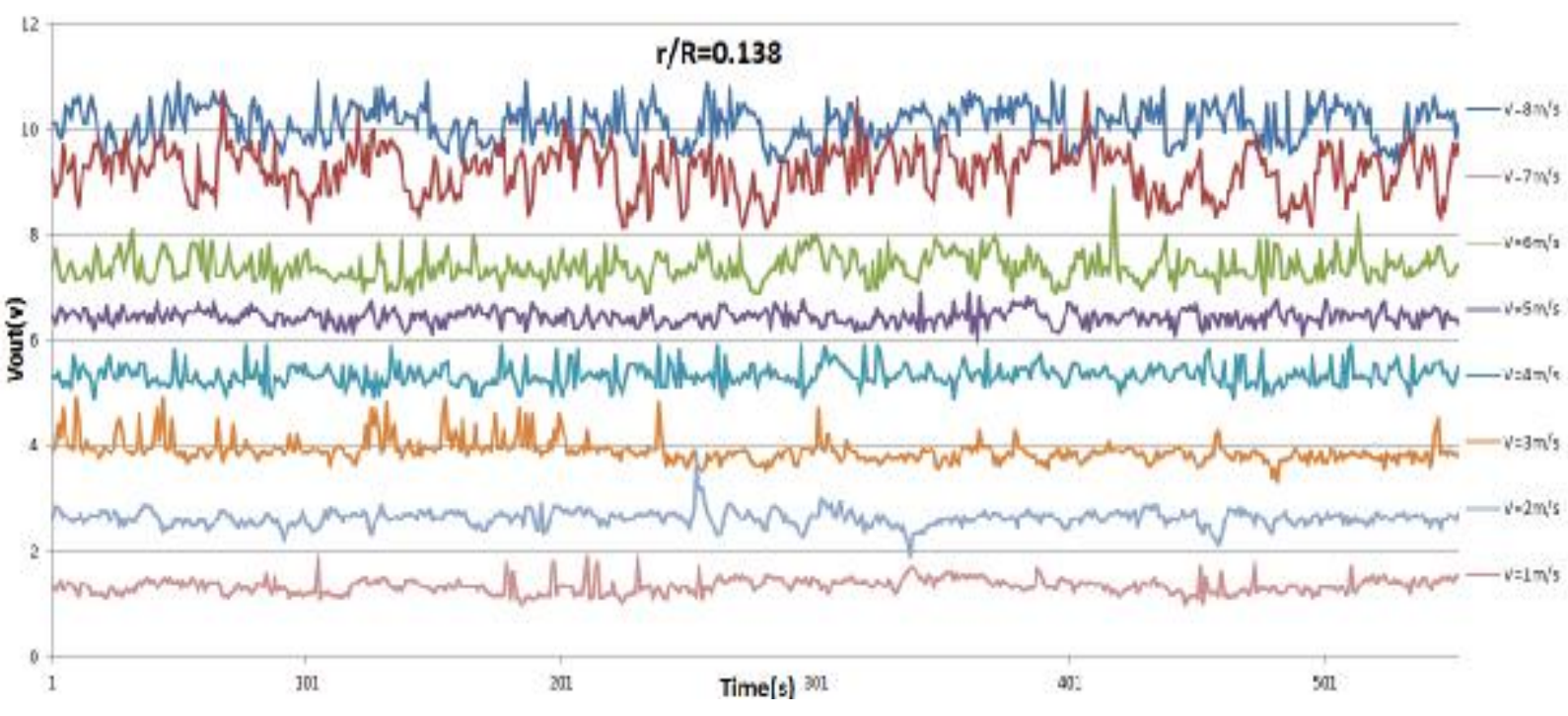

b. $r / R=0.138$

Fig. 3 The velocity measured by HWA for gas flow velocity form $V=1 \mathrm{~m} / \mathrm{s}$ to $8 \mathrm{~m} / \mathrm{s}$ at different $\mathrm{r} / \mathrm{R}$

Figure 4 shows the results of the local averaged velocity for 8 different gas flow velocities. The horizontal and vertical axes are scaled by the dimensionless radius and the measured velocity at different positions along with diameter. In general, the presence of the axial velocity tends to be flatted when the gas velocity increases. But in D325 SRP pipeline, the measured velocity is slightly decreased at $r / R=0.45$, when gas flow velocity is greater than $3 \mathrm{~m} / \mathrm{s}$. This may be related to the reinforced spiral. It is shown that the velocity profile of SRP pipeline is the same as smooth pipeline when the gas flow velocity is less than $3 \mathrm{~m} / \mathrm{s}$. The maximum velocity could occur off the pipe centerline. But when the gas flow velocity is higher than $3 \mathrm{~m} / \mathrm{s}$, the velocity profile of SRP pipeline is changed severely at the position of $r / R=0.45$. The measured velocity here is lower than that of the smooth pipe. But at the center of pipe, the velocity is higher than that of the smooth pipe. This change is shown that theoretical result in smooth pipe couldn't be applied to the pipe of SRP directly. The velocity profile of SRP pipeline is changed, on account of the velocity gradient and vortex effects. This is one of the key reasons why the measurement precision is low. 
The experimental results show that the velocity profile had changed in pipeline of SRP. The velocity distribution in smooth pipeline couldn't fit for the pipeline of SRP. Because of the stiffener and welds of SRP, the velocity gradient is changed rapidly, and the boundary layer near the wall is instable. The instability is changed with flow velocity. The velocity is higher, and the instability is greater.

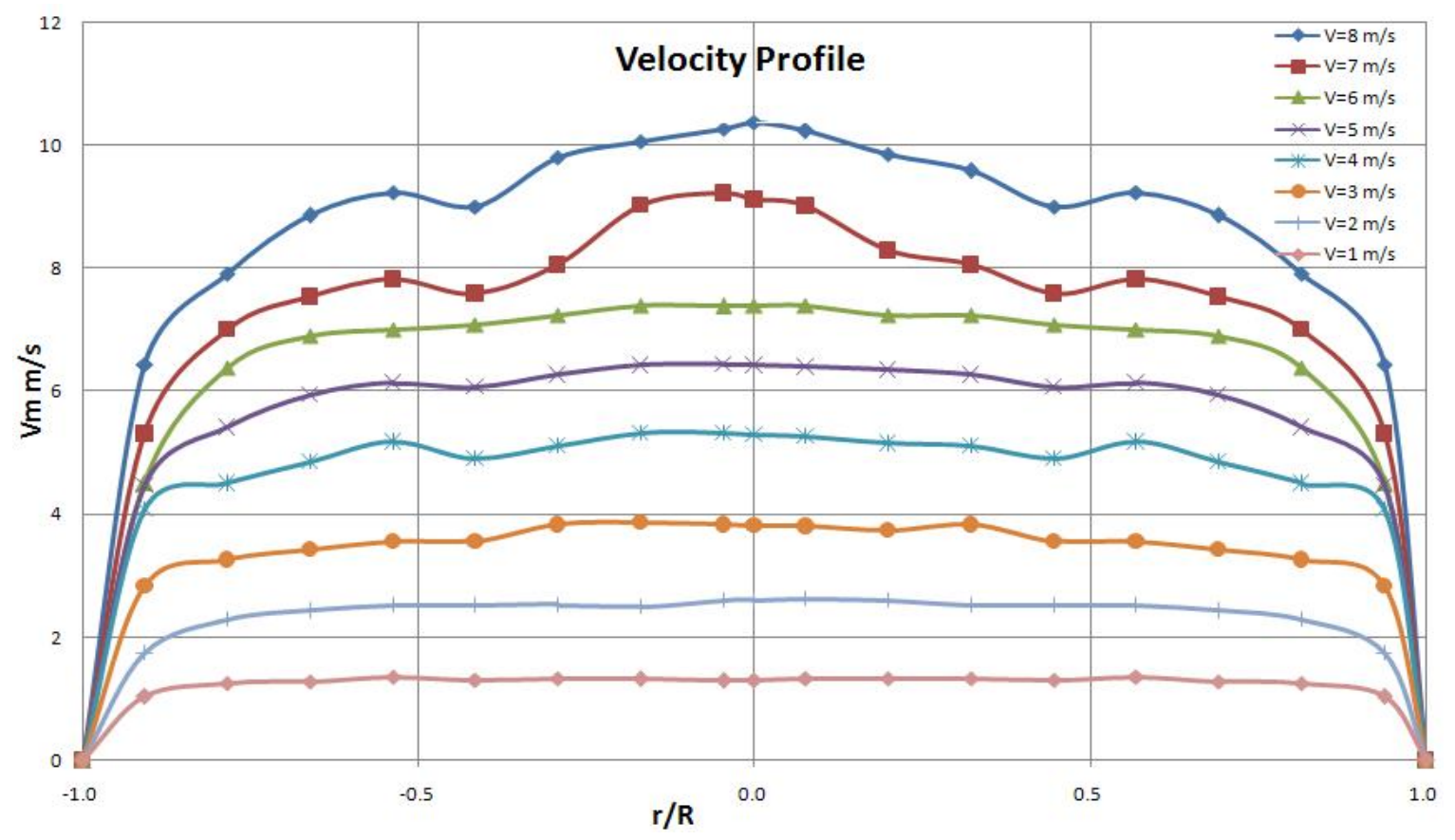

Fig. 4 The velocity distribution in the D325 SRP pipeline.

\section{Conclusion}

The velocity profiles of the large diameter pipe not only depend on the flow velocity, but also depend on the pipe structure. The experimental results show that the turbulence intensity is greater when the flow rate is increased. The velocity distribution profiles change with the structure of the pipe. The measurement results show the maximum gas velocity could occur off the pipe centerline, and a low peak near $\mathrm{r} / \mathrm{R}=0.45$ in $\mathrm{D} 325 \mathrm{SRP}$.

\section{Acknowledgements}

This work was financially supported by the QingkeHengan New Material Limited Company, Tanyuan, Shanxi, 030006, China.

\section{References}

1. Barba, Anna. The prediction of convective heat transfer in spirally fluted tubes.University of Manchester Institute of Science \& Technology, 2012

2. A Barba, AD Gosman, BE Launder.The Prediction of Heat-Transfer Performance in Spirally Fluted Tubes: The Turbulent Flow Regime, 1984

3. JS Yampolsky, PA Libby, BE Launder.Fluid mechanics and heat transfer spirally fluted tubing,Final Report Ga Technologies Inc San Diego Ca,1981

4. V Srinivasan, K Vafai, RN Christensen. Experimental Investigation, Modeling and Prediction of Friction Factors and Friction Increase Ratio for Flow Through Spirally Fluted Tubes, Journal of Enhanced Heat Transfer, 1994 